ESJ Social Sciences

\title{
Solidarité Associative Et Résilience Traumatique : Le Cas De l’Association Sénégalaise Des Victimes De Mines (ASVM)
}

\author{
Ismaïla Sene \\ Laboratoire de Recherche en Sciences Economiques et Sociales (LARSES) \\ Université Assane Seck de Ziguinchor (UASZ) / Sénégal
}

Doi:10.19044/esj.2021.v17n15p142

Submitted: 17 February 2021

Accepted: 06 May 2021

Published: 31 May 2021
Copyright 2021 Author(s)

Under Creative Commons BY-NC-ND

4.0 OPEN ACCESS

Cite As:

Sene I. (2021). Solidarité Associative Et Résilience Traumatique: Le Cas De l'Association Sénégalaise Des Victimes De Mines (ASVM).

European Scientific Journal, ESJ, 17(15), 142. https://doi.org/10.19044/esj.2021.v17n15p142

\section{Résumé}

En Casamance, la multiplication des accidents de mines antipersonnel et antichars dans le contexte du conflit armé a entrainé, chez les victimes, un état de vulnérabilité qui s'est exprimé à travers un affect traumatique. Cette situation a favorisé la mobilisation d'une dynamique d'assistance au centre de laquelle on trouve l'Association Sénégalaise des Victimes de Mines (ASVM). C’est donc le rôle de cette organisation dans la résilience des victimes de mines que cet article se propose d'analyser à travers une méthodologie exclusivement qualitative. Ainsi, grâce à la conduite d'entretiens réalisés avec vingt-sept (27) victimes de mines, deux responsables de l'ASVM et trois responsables au niveau des structures impliquées dans l'assistance aux victimes de mines, l'étude démontre que le recours à la solidarité associative a contribué à l'expression d'un soutien par les pairs. Ce soutien s'est avéré décisif dans la résilience des victimes de mines. Cet article qui se situe à la croisée de la sociologie des organisations et de la psychologie sociale, renouvelle notamment la pensée sur les dynamiques de solidarité associative. Ce faisant, il s'inscrit dans le champ thématique de la sociologie des organisations tout en contribuant à diversifier la réflexion sur le handicap.

Mots-clés: Victimes De Mines, Affect Traumatique, Solidarité Associative, Résilience Traumatique, ASVM, Casamance 


\title{
Associative Solidarity And Traumatic Resilience: The Case Of The Senegalese Association Of Landmine Victims (ASVM)
}

\author{
Ismaïla Sene \\ Laboratoire de Recherche en Sciences Economiques et Sociales (LARSES) \\ Université Assane Seck de Ziguinchor (UASZ) / Sénégal
}

\begin{abstract}
In Casamance, the multiplication of landmine and anti-tank accidents in the context of the armed conflict has led to a state of vulnerability among the victims, which has been expressed through traumatic affect. This situation has led to the mobilization of a dynamic of assistance at the center of which is the Senegalese association of landmine victims (ASVM). It is therefore the role of this organization in the resilience of landmine victims that this article proposes to analyze through an exclusively qualitative methodology. Through interviews with twenty seven (27) landmine victims, two ASVM leaders, and three leaders of structures involved in assisting landmine victims, the study demonstrates that the use of associative solidarity has contributed to the expression of peer support. This support has proven to be decisive in the resilience of landmine victims. This article, which is at the crossroads of organizational sociology and social psychology, renews thinking on the dynamics of associative solidarity. In doing so, it is part of the thematic field of organizational sociology while contributing to the diversification of thinking on disability.
\end{abstract}

Key Words: Landmine Victims, Traumatic Affect, Associative Solidarity, Traumatic Resilience, ASVM, Casamance

\section{Introduction}

En Casamance, l'usage des mines antipersonnel et antichars comme armes de guerre dans le cadre du conflit armé, a favorisé la multiplication des accidents de mines. Ces accidents ont causé, en plus des préjudices physiques (troubles visuels, brûlures corporelles, blessures physiques, amputations des pieds et des mains), de lourds dommages psychologiques (Sène, 2020b). En effet, de par leur caractère brutal et l'étendue des préjudices physiques qu'ils ont causés, ces accidents ont été générateurs d'un affect traumatique qui s’est notamment exprimé, chez les victimes, par un sentiment de rupture identitaire, un sentiment de dégradation et un désespoir. Cet état traumatique a été à l'origine de la mise en œuvre d'une action d'assistance initiée par les pouvoirs publics sénégalais et des acteurs non gouvernementaux afin de réduire la vulnérabilité des victimes de mines (Sène, 2020a). Au cœur de cette dynamique d'assistance, l'Association Sénégalaise des Victimes de Mines 
(ASVM) œuvre dans la réinsertion sociale des victimes de mines à travers l'activation d'une dynamique de solidarité associative. Celle-ci désigne le développement d'une solidarité interne dirigée vers les membres d'une association qui entretiennent entre eux une histoire ou un lien d'identité (Vermeersch, 2001). Ce lien crée ainsi une certaine familiarité entre membres et les amène à se sentir solidaires et à envisager l'entraide mutuellement (Duvoux, 2016). Cette forme de solidarité procède d'une dynamique de création d'un espace de connaissance et de reconnaissance (l'association) qui sert de levier pour développer un élan d'empathie en vers les membres les plus fragiles.

Le présent article s'intéresse à l'enjeu de la solidarité associative développée par l'ASVM dans la prise en charge de l'affect traumatique auquel les victimes de mines ont été confrontées. Il s'inscrit ainsi dans l'ancrage théorique de la psychologie sociale et de la sociologie des organisations. La méthodologie s'appuie sur le recueil de plusieurs témoignages qui rendent compte de la nature de l'affect traumatique tout en mettant en exergue les actions de solidarité entreprises par l'ASVM pour apporter aux victimes de mines un soutien psychosocial porteur de résilience traumatique, laquelle renvoie à la capacité des personnes victimes de traumatisme à s'adapter et à se rétablir avec succès. La présente étude est structurée en trois sections. La première présente le contexte et la problématique de l'étude alors que la deuxième et la troisième section portent successivement sur la méthodologie de recherche et la discussion des résultats.

\section{Contexte et problématique de l'étude}

Depuis qu'il a éclaté en 1982, le conflit armé en Casamance a engendré des conséquences désastreuses sur le plan socioéconomique. Ces conséquences ont été aggravées par la profusion des mines antipersonnel et antichars dont l'usage, comme armes de guerre, a débuté à partir de 1991 (Marut, 2010). Selon une étude sur l'impact des mines en Casamance (Handicap International, 2007), la prolifération des mines a atteint plus de 41 $\%$ du territoire régional de Ziguinchor. Elle a affecté l'activité de plus de 62000 personnes, causant ainsi d'importants préjudices aux plans économique et social.

$\mathrm{Au}$ plan économique, elle a considérablement affecté le développement économique de la Casamance, notamment le secteur agricole (Marut, 2010). En effet, en raison de la présence des mines, les paysans des localités affectées n’ont plus accès ni à leurs rizières ni à leurs plantations fruitières (orangers, anacardiers, citronniers, manguiers, etc.) qui constituaient des ressources importantes pour les familles. Les activités de collecte de bois et de cueillette sont également perturbées (Handicap International, 2007). Aussi, les exploitations horticoles, les forêts et les bois de village se trouvent 
dans un état sauvage, sans entretien et sont en proie aux feux de brousse. Certaines ressources ligneuses et non ligneuses font l'objet d'un pillage systématique. La riziculture est de moins en moins pratiquée par les populations locales qui optent parfois pour l'exode rural. La culture arachidière qui constituait l'une des principales sources de revenus dans la région a également été impactée (Handicap International, 2007). Cette conjoncture a aussi affecté des secteurs tels que la pêche ou encore le tourisme qui a été particulièrement confronté au ralentissement de ses activités (Gueye, 2011). Le secteur du commerce a été également affecté par l'insécurité. Par exemple, le commerce des fruits tels que la mangue et la banane était de plus en plus circonscrit autour des grandes villes (Handicap International, 2007).

Au plan social, on assiste au déplacement de milliers d'individus au fil des années, surtout dans les zones rurales. En raison de l'insécurité liée aux mines et à la présence des forces armées menaçantes, les populations ont été obligées de trouver refuge dans les pays frontaliers et dans les villes de Ziguinchor et Bignona (Robin, 2006). Rien que pour la destination Ziguinchor, le nombre de personnes déplacées internes ayant trouvé refuge dans la ville serait compris entre dix mille (10 000) et quatorze mille (14 000) (IDMC-NRC, 2010).

A côté de ces dommages, la profusion des mines a entrainé un grand nombre de victimes ayant subi des explosions de mines. Ces explosions sont désignées par le terme « accidents de mines » (Sène, 2020a). Avec un total de 751 « victimes directes » (554 blessées et 197 tuées par les mines), le Sénégal a été cité parmi les pays qui enregistrent le plus grand nombre de victimes de mines (CNAMS, 2009). De nos jours, on dénombre plus d'une centaine de survivants qui, du fait de leur handicap, sont confrontés à un cadre de vie sociale et scolaire peu sain et/ou dégradé, à des difficultés d'insertion socioéconomique et aux conséquences psychologiques du traumatisme des mines (Sène, 2020a).

Du fait de cette expérience, les victimes ont été dominées par une fragilité psycho-émotionnelle qui s'est exprimée par une série de réactions négatives comme le stress, l'anxiété, les insomnies, l'irritabilité, la culpabilité ou encore des sentiments de regret (Sène, 2020a). À ces réactions immédiates et parfois temporaires se sont ajoutés, de manière durable, le développement d'un sentiment de dépendance, le désespoir, le sentiment d'infériorité, la perception d'un mépris de la part des proches sans oublier l'émergence de pensées parasites (envisager le suicide comme une solution).

Comme le démontre Sène (2020 a) cette situation de vulnérabilité a été à l'origine de la mise en place de plusieurs mesures d'assistance visant à réparer les préjudices subis par ces victimes et à favoriser, par ricochet, leur épanouissement social. Cette dynamique d'assistance a mobilisé plusieurs catégories d'acteurs dont les plus visibles sont, entre autres, les structures 
étatiques (CNAMS ${ }^{1}$, hôpital Psychiatrique et $\mathrm{CAOSP}^{2}$ ), les organisations non gouvernementales et l'ASVM. Néanmoins, le peu d'attention que la question des mines a suscité de la part des chercheurs qui se sont intéressés à la Casamance n'a pas permis de documenter, de manière suffisante, l'effet de ces acteurs dans l'amélioration des conditions de vie des victimes de mines. Bien que certains travaux aient abordé les conséquences des mines (Marut, 2010 ; Gueye, 2011 ; Sène ; Sène, 2020b) et la question de l'assistance aux victimes de mines (2020a), la problématique des mines et notamment la question de l'assistance aux victimes de mines restent insuffisamment documentées.

Le présent article se propose donc de mettre le focus sur cette question. Il s'inscrit, ainsi, dans la problématique du handicap mais inaugure une nouvelle piste de réflexion qui rompt avec les approches classiques qui ont souvent pensé le handicap à travers les notions de représentations sociales (Chossy, 2011 ; Diop, 2012), d'inclusion scolaire (Ebersold et al., 2006 ; Caraglio, 2017) ou encore d'intégration sociale et/ou professionnelle (Echivard, 2009 ; Weber, 2011 ; Prado, 2014 ; Tonchev, 2014). En effet, la présente étude se propose d'aborder le handicap à travers la relation entre la personne handicapée et son groupe de pairs pour interroger l'enjeu de l'appartenance associative dans la prise en charge du handicap. Une telle rupture procède d'une volonté de penser le handicap dans sa dimension socioidentitaire (enjeu de l'appartenance à un groupe de pairs dans le vécu du handicap) tel que le suggèrent les travaux de Richard (2006) sur la problématique identitaire et les réactions émotionnelles que génère la survenue d'un handicap acquis et la théorie de Bokanowski (2010) sur les implications psycho-émotionnelles négatives du traumatisme.

Par ailleurs, l'accent mis la solidarité associative permet d'appliquer la théorie du "soutien des pairs" (Wallot, 2019) mais aussi celle des " influences sociales des pairs » (Sage et Kindermann, 2000) au contexte associatif pour mettre en évidence la contribution de l'association dans la prise en charge de ses membres. L'analyse de cette "solidarité de proximité » (Balmary, 2015) permet également de mettre en exergue le dépassement de la logique classique de prise en charge de la vulnérabilité basée sur la solidarité institutionnelle. En effet, de par sa rencontre quotidienne avec les membres fragiles, elle est en capacité de combler les lacunes de la solidarité institutionnelle car étant apte à répondre à une demande sociale qui s'inscrit de moins en moins dans les catégories juridiques traditionnelles du droit social, mais qui réclame une intervention de plus en plus personnalisée, complexe et humaine (Balmary, 2015).

${ }^{1}$ Centre National d'Action Antimines au Sénégal.

${ }^{2}$ Centre Académique de l'Orientation scolaire et professionnelle de Ziguinchor. 
Dès lors, étant l'unique association de victimes de mines en Casamance et développant un soutien exclusivement orienté vers ses membres associés, l'ASVM constitue naturellement la cible de cette étude qui interroge l'enjeu de la solidarité associative dans la résilience des victimes de mines. Créée en 1999, pendant une période marquée par une forte recrudescence des accidents de mines en Casamance, l'ASVM est une association de droit sénégalais qui regroupe les victimes civiles de mines (antipersonnel et antichars) du conflit en Casamance. Son objectif est de contribuer à la réinsertion sociale et économique de ses membres en créant un environnement favorable à leur épanouissement par le biais notamment d'une assistance sanitaire, sociale, économique et parfois scolaire. Elle développe ainsi une solidarité associative orientée vers ses membres, les plus fragiles notamment, pour favoriser leur résilience à la suite du traumatisme vécu. C'est donc l'enjeu de cette solidarité dans la résilience des victimes de mines que la présente étude se propose d'analyser.

Ce faisant, la problématique s'articule autour de la question ci-après : quel est l'enjeu de solidarité associative, développée par l'ASVM, dans la résilience traumatique des victimes de mines en Casamance?

\section{Méthodologie de recherche}

Cette étude s'est appuyée sur la méthode qualitative. Les données ont été recueillies grâce à une série d'entretiens semi directifs et de récits de vie réalisés (entre janvier et avril 2018) avec vingt-sept (27) victimes de mines. Les entretiens ont duré entre 30 et 35 minutes en moyenne. Toutefois, les rencontres répétées avec les victimes ont permis d'établir une relation de confiance avec elles et d'inscrire l'approche méthodologique dans une démarche itérative. Cette démarche a permis notamment de recueillir des témoignages des victimes vivant dans la région de Ziguinchor, principal foyer du conflit en Casamance et qui abrite l'écrasante majorité de la population de victimes de mines ${ }^{3}$. Des entretiens ont été également effectués avec trois responsables travaillant au niveau des structures impliquées dans la prise en charge psychosociale des victimes de mines (CNAMS, CAOSP et Hôpital psychiatrique de Ziguinchor).

Ainsi, grâce à une approche alliant diversification et recherche de la saturation empirique, nous avons pu discuter des implications psychotraumatiques du handicap lié aux mines mais aussi et surtout des actions de solidarité développées par l'ASVM pour contribuer à la résilience des victimes de mines. A l'aide d'un guide d'entretien semi-structuré, les victimes de mines ont été notamment amenées à répondre aux questions ci-après : Comment

\footnotetext{
${ }^{3}$ Un recensement effectué par le CNAMS (2009) fait état de 151 victimes directes vivant dans la région de Ziguinchor sur un total de 182 recensées en Casamance (soit un taux 82,96 $\%)$.
} 
avez-vous vécu votre handicap ? Quelles sont les actions de solidarité développées par l'ASVM à votre encontre ? Quels sont, pour vous, les effets des actions d'assistance développées par l'ASVM ? Comment appréciez-vous les efforts consentis par l'ASVM pour accompagner les victimes de mines ?... Pour leur part, les responsables de l'ASVM ont été interrogés sur les actions d'assistance qu'ils ont développées à l'endroit des victimes de mines, le modèle d'intervention utilisé, le ressenti des victimes ou encore l'effet de leurs actions dans l'amélioration du vécu du handicap. Enfin, les responsables des structures d'accompagnement ont été notamment interrogés sur les causes et les enjeux de la collaboration avec l'ASVM.

Les diverses informations recueillies ont été exploitées grâce à une analyse de contenu qui a notamment mis en exergue les variables relatives à l'affect traumatique chez les victimes de mines et celles traitant de l'effet de l'ASVM dans la prise en charge de cet affect.

\section{Résultats et discussion}

Cette partie décrit d'abord la nature de l'affect traumatique vécu par les victimes de mines avant d'analyser la contribution de l'ASVM dans la résilience de ces dernières.

\section{Affect traumatique des victimes de mines}

Comme l’ont démontré de précédentes études (Sène, 2020a ; Sène, 2020b), les accidents de mines sont à l'origine de plusieurs formes de réactions émotionnelles immédiates à l'exemple des sentiments de rupture identitaire, de dégradation et de désespoir qui ont été constatés chez plusieurs victimes.

De telles réactions renseignent sur les difficultés d'intégration de l'évènement traumatique par les victimes. Elles renseignent également sur les problèmes d'acceptation de la situation de handicap acquis du fait de la présence des mines. En effet, dans le récit de l'histoire traumatique des victimes, on s'aperçoit que la perte d'un membre est apparue comme un élément déterminant de l'affect, lequel a été marqué par l'apparition de plusieurs émotions négatives. Selon les témoignages recueillis le stress était l'émotion la plus représentative car la majeure partie des victimes a décrit le stress comme étant la principale réaction dans le contexte post-accident. Pour d'autres, la réaction dominante était la colère ou l'angoisse. Le témoignage de F.T. (une victime de mine) permet de mieux comprendre cette situation :

"J'étais trop affectée par mon handicap car je ne me suis jamais imaginée vivre avec un pied amputé. A un moment j'en voulais à tout le monde même à moi-même. J'étais trop stressée et irritable. Jusqu'à présent, et sans les connaitre, je continue à détester ceux qui ont placé la mine dans cet endroit. Je pense que depuis cet évènement, je suis devenue 
trop nerveuse et très susceptible. Parfois je sens une peur m'envahir sans que je ne sache d'où ça vient mais je sais qu'elle a un lien avec cet événement ».

De manière générale, cet état d'alerte traumatique s'est notamment exprimé par des cauchemars répétitifs, la peur excessive et des insomnies sévères. L'apparition de pensées " parasites " constitue également un dommage collatéral. Plus d'un tiers des victimes affirment avoir pensé à se suicider après avoir pris conscience du handicap. L'exemple de C.G (une victime de mine) démontre bien la lourdeur de l'affect :

"Dans les jours qui ont suivi l'accident, mon principal problème était l'angoisse. J'ai beaucoup pensé à ma situation d'avant et en me regardant, je me disais dans ma tête que j'aurais dû éviter l'accident. Je ne sais pas comment mais c'est ce que je me disais (...). J'ai eu du mal à accepter ma situation car, à mes yeux, j'étais quelqu'un d'autre ».

Cet exemple, comme tant d'autres, permet de comprendre l'accident de mine comme une expérience traumatisante qui a bouleversé la structure cognitive et le schéma émotionnel des victimes de mines. En effet, les émotions et sentiments développés par ces dernières, à la suite de l'accident, démontrent les difficultés d'acceptation et d'intégration du handicap dans leur schéma cognitif. Cet état de fait reste lié au sentiment de rupture identitaire que provoque le handicap acquis. Comme l'a démontré Boquel (2009), cette rupture ébranle la cuirasse caractérielle de la victime et provoque une "impasse événementielle d'origine traumatique " (Boquel, 2009).

Chez la plupart des victimes, cette impasse s'est notamment exprimée en termes de comparaison entre le moi ancien et le moi actuel ; le premier étant perçu comme le moi idéal alors que l'autre est vu comme dégradant. Les propos de F.B. (une victime) illustrent bien cet état de fait :

"A chaque fois que je pense à ma situation d'avant, je suis triste. J'étais mobile et très autonome. Actuellement, je suis condamné à rester sur un fauteuil car je ne peux rien pour moi-même. C'est difficile à accepter mais je me sens inférieur ».

Comme on peut bien s'en apercevoir, la comparaison identitaire débouche souvent sur le développement d'un sentiment d'impuissance et la persistance d'une image cognitive qui remettent en question le sentiment d'auto-efficacité et qui démontrent les difficultés à surmonter le handicap acquis. Cela a entraîné, chez certaines victimes, le développement d'un sentiment d'inutilité 
sociale mais aussi la perception d'une certaine forme d'altérité qui pousse la personne «nouvellement» handicapée à se percevoir comme différente voire anormale. Le contraste entre l'état de validité physique antérieur et la "nouvelle " situation de handicap a donc entraîné un problème d'acceptation identitaire chez des victimes qui, vivant avec un « avant et un après » (Richard, 2006), ont éprouvé des difficultés à faire le deuil de leur handicap.

Cet état affectif délicat a donc favorisé la mobilisation d'une solidarité associative qui s'est développée autour de l’ASVM.

\section{Effet de l'ASVM dans la résilience des victimes de mines}

Fort de son objectif de contribuer à la réinsertion sociale et à l'épanouissement de ses membres, l'ASVM s'est positionnée comme un acteur-clé dans la conduite de la politique d'assistance initiée en faveur des victimes de mines en Casamance. En effet, en faveur de ces dernières, elle intervient, entre autres, dans la conduite des activités de rééducation physique, la prise en charge médicale et l'aide à l'emploi. A travers ces piliers de la politique d'assistance aux victimes, elle facilite, par le biais d'un travail d'intermédiation associative, le ciblage, l'expression des besoins et la mobilisation des bénéficiaires. Cette association constitue donc un intermédiaire par qui passent les structures impliquées dans l'assistance aux victimes de mines. Comme l'explique un responsable du Centre National d'Action Antimines au Sénégal (CNAMS) :

«L'ASVM est le seul répondant des victimes de mines. Nous nous appuyons beaucoup sur ses responsables pour rendre opérationnelles les actions proposées. Nous travaillons aussi avec eux pour définir le domaine d'intervention et l'orientation des actions ».

Il convient de noter que c'est notamment dans le domaine de l'assistance psychologique que la portée de l'action de l'ASVM semble beaucoup plus visible. En effet, dans le cadre de leur intervention, les structures d'écoute et de prise en charge psychologique ${ }^{4}$ s'appuient sur les membres de cette association pour conduire des activités d'assistance psychologique. C'est pour cette raison qu'elle est citée, à côté de ces structures, comme étant une actrice majeure de cette forme d'assistance. En effet, elle constitue généralement le bras technique de ces structures dont les spécialistes forment ses membres sur la conduite du débriefing psychologique et

${ }^{4}$ Centre Académique de l'Orientation scolaire et professionnelle de Ziguinchor (CAOSP) et Hôpital psychiatrique de Ziguinchor. 
de l'écoute empathique pour leur permettre de développer ces actions auprès des victimes de mines.

Cette démarche répond à une volonté de faciliter l'accès des cibles aux services d'assistance psychologique. En effet, l'insuffisance des ressources humaines au niveau de ces structures et l'inaccessibilité de certaines zones obligent ces dernières à s'appuyer sur les leaders de l'association et ses responsables de sections (départementales et communales) pour rendre effectif le soutien psychosocial avec toutes ces exigences en termes de proximité et de durabilité. A ce propos, un responsable du CAOSP (structure impliquée dans la conduite de cette forme d'assistance) estime que :

"Le CAOSP collabore souvent avec les responsables de l'ASVM pour assurer aux victimes nécessiteuses un suivi régulier. On s'appuie sur eux car ils ont vécu les mêmes expériences. Mais auparavant, on les capacite pour les aider à mieux conduire des séances de débriefings collectifs. Ils animent aussi des séances de sensibilisation et mènent d'autres activités de soutien parallèle ».

Pour sa part, le témoignage du Directeur de l'Hôpital psychiatrique (autre structure impliquée dans l'assistance psychosociale aux victimes de mines) permet de mieux comprendre l'enjeu de cette approche :

"On a compris que ces leaders associatifs étaient incontournables pour l'efficacité de l'aide psychologique. Leur implication nous permet de régler le problème d'accessibilité. C'est l'association qui connait mieux les victimes, elle facilite donc le repérage et ses membres sont formés pour dérouler des débriefings collectifs dans la phase de rééducation psychologique ».

Néanmoins, au-delà de l'accessibilité, il faut souligner que cette implication permet d'améliorer la qualité du soutien psychologique offert aux victimes de mines. En effet, la proximité identitaire entre les victimes et les responsables de l'ASVM (euxmêmes victimes de mines) facilite la compréhension mutuelle et l'entraide. Comme le suggèrent Jaouen et al. (2006), une relation d'accompagnement (d'assistance psychologique) combine technicité, empathie et disponibilité. Ainsi, dès lors qu'ils bénéficient d'un renforcement de capacités, les responsables de l'ASVM se positionnent comme les seuls acteurs capables de prendre en compte ces trois enjeux dans le temps. Comme le soutient B.G. (une victime de mine) ;

"L'ASVM est très proche des victimes. Dès qu'un accident est déclaré, ils sont les premiers à se présenter pour soutenir 
et assister la victime. Ils viennent souvent à notre chevet car ils nous comprennent. Ils sont des victimes, ils ont vécu pareilles situations c'est pourquoi, ils veillent sur nous pour nous éviter de nous sentir seul ».

Par ailleurs, ces derniers organisent également des visites à domicile pour écouter les victimes afin de leur apporter un soutien social face à des difficultés d'acceptation ou de compréhension dont elles sont parfois victimes de la part de leur entourage. Ces visites ont souvent débouché sur des actions de médiation familiale pour expliquer aux proches la nécessité de développer un soutien social empreint d'empathie et d'éviter toute attitude pouvant entrainer, chez les victimes, la perception d'une forme de stigmatisation quelconque. A l'occasion d'un entretien ténu avec lui, le président de l'ASVM a bien insisté sur cet aspect :

"On a réglé beaucoup d'incompréhensions entre des victimes et leurs proches. Parfois, les familles communiquent mal avec les victimes. Aussi, elles ne savent pas traiter avec les victimes. C'est pourquoi leurs attitudes sont souvent maladroites et les victimes les interprètent de manière négative. Vivre avec un handicap n'est pas facile, la perte d'un membre entraine des effets négatifs. Chacun a sa manière de gérer ça. Certaines victimes sont très nerveuses et facilement irritables. C'est pourquoi, les visites en milieu familial nous permettent de faciliter la communication entre eux et d'apporter le soutien nécessaire aux victimes. Nous travaillons également à leur redonner espoir car à travers nous, elles peuvent facilement comprendre qu'elles ne sont pas seules. On essaye également de les amener à vivre leur handicap de manière positive. On a vécu la même expérience, donc c'est très facile de se comprendre entre nous ».

En s'appuyant sur la proximité identitaire, les membres de l'association facilitent l'échange avec les victimes de mines et contribuent à réduire la vulnérabilité de ces dernières en les amenant à comprendre qu'elles ne sont pas seules dans leur souffrance. Cette approche permet également de rétablir le lien social rompu ou devenu vulnérable avec le handicap. C'est ce que laissent paraitre les propos de K.D. (une victime de mine) :

"Personnellement, si j'ai pu sentir une certaine amélioration dans ma situation c'est grâce aux leaders de l'association. Ils nous apportent un soutien que nos proches ne nous apportent même pas. Pour ma part, je peux dire que 
c'est grâce à eux que ma famille me considère désormais. Au début, j'étais vraiment incomprise mais depuis qu'ils sont venus échanger avec ma famille, je me sens moins rejetée ».

La contribution de l'organisation dans la réinsertion sociale des victimes de mines s'aperçoit, en outre, à travers l'organisation de rencontres collectives qui ont favorisé le soutien des pairs. En effet, le pari de faciliter l'inclusion sociale, à partir de la solidarité associative, a permis à l'ASVM d'aider certaines victimes à s'identifier à d'autres et à s'assister mutuellement. Les enquêtes effectuées ont permis de se rendre compte que plusieurs victimes ont commencé à relativiser leur situation à la suite des rencontres d'échanges organisées par l'association. L'exemple de M.B. (une victime) permet d'illustrer ce constat :

« Je m'étais isolée et je passais tout mon temps à m'apitoyer sur mon sort. Quand je suis allée à une réunion des victimes, j'ai rencontré des gens qui avaient des préjudices beaucoup plus sévères. Certains avaient des doubles amputations. J'ai vu quelqu'un qui était amputé du pied et de la main. C'est en voyant ces gens que j'ai compris qu'il y avait d'autres personnes qui vivaient une situation beaucoup plus délicate. C'est en ce moment-là que j'ai commencé à accepter ma situation. C'est de là-bas que j'ai recommencé à discuter et à m'ouvrir aux autres car je me disais parfois que j'ai été chanceuse de me retrouver avec un seul pied amputé ».

On s'aperçoit à travers les propos de cette victime (et à travers ceux d'autres victimes) de l'efficacité des échanges avec les groupes de pairs. Les rencontres de groupes sont, en effet, des occasions de partage et d'écoute qui aident certaines victimes à sortir de leur angoisse personnelle et à s'identifier à des personnes qui partagent leur vécu. L’appartenance ou même la participation à un groupe de pairs permet ainsi de minimiser certaines souffrances qui découlent d'un repli sur soi et de l'existence d'un rejet familial dont bon nombre de handicapés croient être victimes. En témoignent les propos de M.N (une victime de mine) :

"Les réunions sont très utiles. On se conseille mutuellement et cela crée une proximité. Cela nous amène à dédramatiser notre situation et mieux nous affirmer. (...). J'apprécie vraiment la solidarité qui prévaut ici. Je sais que sans l'ASVM les victimes seraient dispersées et vivraient dans l'oubli. Aujourd'hui beaucoup de projets s'intéressent à nous grâce aux responsables de l'ASVM. Ils collaborent 
avec beaucoup d'institutions pour nous aider dans la formation, l'entrepreneuriat et surtout l'intégration sociale. Ils ne peuvent pas faire tout mais ils font de leur mieux. On est fier d'eux ».

Il va s'en dire que l'organisation de ces rencontres a favorisé l'apparition d'une identité de groupe au moment où certaines victimes se sont effectivement rendu compte qu'elles partageaient leur vécu avec d'autres. Ces rencontres ont également permis à d'autres victimes de se départir du complexe lié au handicap acquis, cela du fait du contact avec des pairs qui vivaient une situation beaucoup plus dramatique.

Cette manière d'être soutenu par les pairs témoigne non seulement d'un processus relativement inversé à la logique classique de l'accompagnement (Jaouen et al., 2006) mais aussi d'une relation d'échange où se crée une situation de gagnant-gagnant entre la victime et les responsables de l'organisation qui, eux aussi, voient à travers les victimes leurs semblables. Ce qui donne ainsi naissance à une sorte d'alliance stratégique et renforce les performances sociales de l'organisation.

\section{Conclusion}

En définitive, l'étude démontre que les membres de l'ASVM ont développé plusieurs actions qui ont concouru à réduire la vulnérabilité des victimes de mines par le biais d'une résilience associative. Elle met ainsi en exergue l'enjeu de la solidarité associative dans la résilience des membres. Elle démontre ainsi que le soutien des pairs constitue une logique de solidarité associative qui contribue à réduire la vulnérabilité des membres dans un contexte où les spécialistes de la relation d'aide peinent à apporter toute l'assistance requise. Cette étude renforce ainsi la littérature sur les organisations associatives et notamment sur l'analyse des dynamiques de solidarités qui se déploient au sein de ce type d'organisation. Elle constitue également une contribution significative à la réflexion sur le handicap.

Toutefois, l'une des questions qu'il faudrait se poser à la suite des constats préalablement établis c'est: quelles autres activités l'ASVM développe-t-elle pour inscrire la résilience des victimes de mines dans la durée ? En plus de mettre en exergue un des aspects non couverts par la recherche, cette question ouvre, pour ainsi dire, une piste de réflexion qui se présente comme une des perspectives de la présente étude.

\section{References:}

1. Balmary, D. (2015). L’action associative face aux défis de la solidarité, C.E.R.A.S, Revue Projet, $\mathrm{n}^{\circ}$ 346, pp. 42-49. URL : https://www.cairn.info/revue-projet-2015-3-page-42.htm. 
2. Bokanowski, T. (2010). « Du traumatisme au trauma : les déclinaisons cliniques du traumatisme en psychanalyse », Revue Psychologie clinique et projective, $\mathrm{n}^{\circ}$ 16, 9-27.URL : https://www.carin.info/revuepschologie-clinique-et-projeective-2010-1-page-9.htm.

3. Boquel, P. (2009). Handicap, traumatisme et impasse. Paris, Editions EDK.

4. Caraglio, M. (2017). Les élèves en situation de handicap : inclusion, encore un effort. Actes de communication, 39e colloque de l'AFAE. URL:https://cache.media.eduscol.education.fr/file/Formation_continu e_enseignants/97/3/Les_eleves_en_situation_de_handicapM._Caraglio_au_colloque_AFAE_978973.pdf.

5. Chossy, J.F. (2011). Evolution des mentalités et changement du regard de la société sur les personnes handicapées : passer de la prise en charge à la prise en compte. Paris, La Documentation française.

6. CNAMS. (2009). Plan d'Action National pour l'Assistance aux Victimes 2010-2014. Rapport de planification. Centre National D’action Antimines au Sénégal.

7. Diop, I. (2012). Handicap et représentation sociales en Afrique occidentale. Revue le Français aujourd'hui, n¹77, 19-27. URL : https://www.carin.info/revue-le-francais-aujourd'hui-2012-2-page19.htm.

8. Duvoux, N. (2016). La solidarité sociale n'est pas un sentiment, mais cette réalité de l'interdépendance entre les membres de la société. Visions solidaires pour demain. URL : www.solidarum.org.

9. Ebersold, S., Plaisance, E., et Zander, C. (2006). Ecole inclusive pour les élèves en situation de handicap : accessibilité, réussite scolaire et parcours individuels. Rapport de recherche CNESCO, halshs01445378.

10. Echivard, J. P. (2009). Représentations du handicap et pratiques d'accompagnement. Revue Empan, $\mathrm{n}^{\circ} 74$, 45-49. URL : https://www.carin.info/revue-empan-2009-2-page-45.htm.

11. Gueye, D. (2011). Résultats de l'étude des Connaissances-AptitudesPratiques (CAP). Rapport d'étude. CNAMS, ASVM, HI, UNICEF.

12. Handicap International. (2007). Etude d'urgence sur l'impact des mines en Casamance. Rapport de recherche. URL : http://www.franco-mines.com.

13. IDMC-NRC. (2010). Sénégal. De nouveaux déplacements et défis à l'obtention de solutions durables en Casamance. Rapport d'étude.

14. Jaouen, A., Loup S., et Sammu S. (2006). Accompagnement par les pairs, confiance partagée et résilience : illustration au travers du cas voiles d'OC. De Boeck Supérieur | Revue de l'Entrepreneuriat, ${ }^{\circ} 1$ Vol. 5, pp. 59 - 72. 
15. Manga, M.L. (2014). La Casamance dans l'histoire contemporaine du Sénégal, Paris Harmattan.

16. Marut, J. C. (2010). Le conflit de Casamance : ce que disent les armes. Paris, Karthala.

17. Nelly, R. (2006). « Le déracinement des populations en Casamance : Un défi pour l'État de droit ", Revue européenne des migrations internationales, vol 22, $\mathrm{n}^{\circ} 1$, pp. 153-181.

18. Prado, C., (2014). Mieux accompagner et inclure les personnes en situation de handicap : un défi, une nécessité. Paris, Les éditions des journaux officiels.

19. Richard, J.T. (2006). Psychanalyse et Handicap. Paris, Harmattan.

20. Sage, N. A. et Kindermann, T. A. (2000). «Influences sociostructurelles du groupe de pairs sur la motivation scolaire des jeunes enfants ». Revue des sciences de l'éducation, 26 (1), 133-150. URL : https://doi.org/10.7202/032031ar.

21. Sène, I. (2020 a). Le conflit casamançais : focus sur l'assistance aux victimes de mines. Paris, Harmattan.

22. Sène, I. (2020 b). Handicap acquis, affect traumatique et perception de soi : Le cas des victimes de mines en Casamance. Revue internationale des sciences économiques et sociales. Paris, Harmattan, pp.121-138.

23. Tonchev D. (2014). Les politiques du handicap. Paris, Editions ASPH.

24. Vermeersch, S. (2001). L'engagement associatif : quelles solidarités?. Les Annales de la recherche urbaine, $N^{\circ} 89$, pp. 46-52.

25. Wallot, H.A. (2019). Le soutien des pairs souffrant de psychose : l'expérience du programme "Passer l'espoir au suivant ». Santé mentale au Québec, 44(1), pp. 111-126. URL : https://doi.org/10.7202/1060279ar.

26. Weber, F. (2011). Handicap et dépendance : Drames humain, enjeux politiques. Paris, Editions Rue d'ULM. 